Asia Pac. J. Math. 2022 9:6

ASIA PACTFIC ACADEMIC

\title{
ON SIMPLE POLYNOMIAL BOUNDS FOR THE EXPONENTIAL FUNCTION
}

\author{
CHRISTOPHE CHESNEAU ${ }^{1}$, YOGESH J. BAGUL ${ }^{2, *}$, RAMKRISHNA M. DHAIGUDE $^{3}$ \\ ${ }^{1}$ Université de Caen Normandie, LMNO, Campus II, Science 3, 14032, Caen, France \\ ${ }^{2}$ Department of Mathematics, K. K. M. College, Manwath, Dist: Parbhani(M. S.)-431505, India \\ ${ }^{3}$ Department of Mathematics, Government Vidarbha Institute of Science and Humanities,
} Amravati(M.S.)-444604, India

*Corresponding author: yjbagul@gmail.com

Received Jan. 3, 2022

\begin{abstract}
Авstract. In this article, we offer a new polynomial or polynomial-exponential bounds for the exponential function. Its main interest is to be both simple and sharp, under some clear conditions on the parameters involved. Applications are given for a probability function and the Kummer beta function.
\end{abstract}

2010 Mathematics Subject Classification. 26D07, 33B10, 33B20.

Key words and phrases. exponential inequality; productlog function; normal distribution; Kummer beta function.

\section{INTRODUCTION}

The natural exponential functions are extremely important in many branches of science and mathematics. Sometimes we require the bounds of such a function on the interval $[0,1]$ for a specific purpose. One obvious upper bound is given in the following inequality: For any $x \in[0,1)$,

$$
e^{x} \leq \frac{1}{1-x}
$$

The inequality (1) is coarser and its refinement is given in [3]. For some other sharp bounds, we refer to $[1,3]$ and the references therein. The bounds in the present literature are somewhat complex in nature; there is still a need for tractable and sharp bounds in all branches of

DOI: 10.28924/APJM/9-6

(C2022 Asia Pacific Journal of Mathematics 
mathematics. The main goal of this paper is to achieve useful and simpler polynomial or polynomial-exponential bounds for exponential functions than those available in the literature.

\section{Main Result}

The following result presents the main finding of the study.

Proposition 1. For any $x \in[0,1]$ and $a \in \mathbb{R}$, we have

$$
\operatorname{sign}(a) e^{a x} \leq \operatorname{sign}(a)\left[a x(1-x)+x^{2}\left(e^{a}-1\right)+1\right]
$$

where $\operatorname{sign}(a)=-1$ if $a<0, \operatorname{sign}(0)=0$ and $\operatorname{sign}(a)=1$ for $a>0$.

Proof. We propose a proof based on the analysis of appropriate functions. To begin, let us consider the following function:

$$
f(x ; a)=e^{a x}-a x(1-x)-x^{2}\left(e^{a}-1\right)-1 .
$$

Then, upon differentiation, we have

$$
f^{\prime}(x ; a)=a e^{a x}+2\left(a-e^{a}+1\right) x-a .
$$

The equation $f^{\prime}(x ; a)=0$ has two solutions only into $[0,1]$, which are $x_{0}=0$ and $x_{1}=$ $\left[y_{a}-W\left(y_{a} e^{y_{a}}\right)\right] / a$, where $y_{a}=a^{2} /\left[2\left(a+1-e^{a}\right)\right]$ and $W(x)$ is the productlog function defined by $W(x) e^{W(x)}=x$. Clearly, the nature of $x_{0}$ and $x_{1}$ are informative on the possible sign of $f(x ; a)$. Since $f(x ; a)$ is continuous with $f(0 ; a)=0$ and $f(1 ; a)=0$, it is enough to study the nature of the extremum $x_{0}$; if $x_{0}$ a local minimum, then $x_{1}$ is a local maximum, and vice versa.

First, let us notice that

$$
f^{\prime \prime}(x ; a)=a^{2} e^{a x}+2\left(a-e^{a}+1\right),
$$

which implies that

$$
f^{\prime \prime}(0 ; a)=a^{2}+2\left(a-e^{a}+1\right)=2 \phi(a)
$$

where

$$
\phi(a)=1+a+\frac{a^{2}}{2}-e^{a} .
$$

Since $e^{a}>1+a$ for any $a \in \mathbb{R}^{*}$, we have $\phi^{\prime}(a)=1+a-e^{a}<0$, which implies that $\phi(a)$ is decreasing for any $a \in \mathbb{R}$. Let us now study the nature of the extremum $x=0$ according to $a>0$ and $a<0$ via the second derivative test. 
- For $a>0$, we have $\phi(a)<\phi(0)=0$, implying that $f^{\prime \prime}\left(x_{0} ; a\right)=f^{\prime \prime}(0 ; a)<0$. Thus, $x_{0}$ is a local maximum point, implying that $x_{1}$ is necessary a local minimum point: For any $x \in[0,1]$ and $a>0$, we have

$$
f(x ; a) \leq \min (f(0 ; a), f(1 ; a))=0,
$$

which implies the desired inequality.

- For $a<0$, we have $\phi(a)>\phi(0)=0$, implying that $f^{\prime \prime}\left(x_{0} ; a\right)=f^{\prime \prime}(0 ; a)>0$. Thus, $x_{0}$ is a local minimum point, implying that $x_{1}$ is necessary a local maximum point: For any $x \in[0,1]$ and $a<0$, we have

$$
f(x ; a) \geq \max (f(0 ; a), f(1 ; a))=0,
$$

which implies the desired inequality.

This ends the proof.

Remark 1. For the case $a>0$, some alternative proofs can be given. By the series expansion of the exponential function, since $x \in[0,1]$, we have

$$
\begin{aligned}
e^{a x}-1 & =\sum_{k=1}^{+\infty} \frac{(a x)^{k}}{k !}=a x+\sum_{k=2}^{+\infty} \frac{(a x)^{k}}{k !} \leq a x+x^{2} \sum_{k=2}^{+\infty} \frac{a^{k}}{k !} \\
& =a x+x^{2}\left(e^{a}-1-a\right)=a x(1-x)+x^{2}\left(e^{a}-1\right) .
\end{aligned}
$$

Remark 2. For the case $a<0$ and $x \in[0,1]$, the proposed lower bound improves the famous inequality $e^{a x} \geq 1+a x$. Indeed, by using $e^{a} \geq 1+a$, we have

$$
e^{a x} \geq a x(1-x)+x^{2}\left(e^{a}-1\right)+1=1+a x+x^{2}\left(e^{a}-1-a\right) \geq 1+a x .
$$

Remark 3. Proposition 1 can be extended to any bounded interval of the form $[0, c]$ for $x$, with $c>0$. In this case, it is enough to replace $x$ by $x / c$. That is, for any $x \in[0, c]$, since $x / c \in[0,1]$, we have

$$
\operatorname{sign}(a) e^{a x / c} \leq \operatorname{sign}(a) \frac{1}{c^{2}}\left[a x(c-x)+x^{2}\left(e^{a}-1\right)+c^{2}\right],
$$

Remark 4. For $x \in[0,1]$ and $a=x$ or $a=-x$, Proposition 1 yields simple polynomial bounds for $e^{x^{2}}$ and $e^{-x^{2}}$, respectively. More precisely, we have:

$$
e^{x^{2}} \leq x^{2}(1-x)+x^{2}\left(e^{x}-1\right)+1
$$

and

$$
-x^{2}(1-x)+x^{2}\left(e^{-x}-1\right)+1 \leq e^{-x^{2}}
$$


The functions $e^{x^{2}}$ and $e^{-x^{2}}$ are involved in a plethora of mathematical and physical quantities, more or less complex. Our bounds can be of interest for direct bounds of these quantities. See, for instance, [2].

A graphical illustration of Proposition 1 is given in Figure 1. It shows the curve of the function $f(x ; a)$ defined by (2) for some values of $a>0$ and $a<0$.

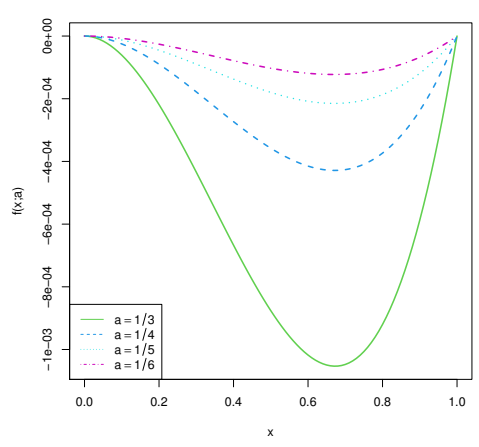

(a)

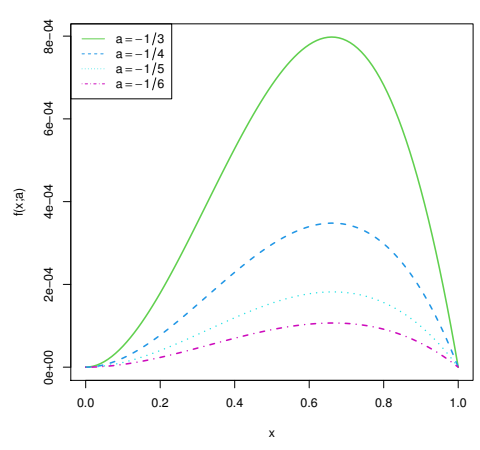

(b)

Figure 1. Curves of $f(x ; a)$ as defined by (2) for $x \in[0,1]$ and (a) some values of $a>0$ and (b) some values of $a<0$.

In Figure 1, we clearly identify the extrema $x_{0}$ and $x_{1}$ as described in the proof of Proposition 1. Also, the sharpness of the obtained bounds can be observed; the maximum of magnitude being between $10^{-4}$ and $10^{-3}$ for the considered values of $a$.

\section{Applications}

Two applications of Proposition 1 are examined in this section.

3.1. Bound of a useful probability. Let $X$ be a random variable with the standard normal distribution, i.e., with probability density function $f(x)=1 /(2 \pi)^{-1 / 2} e^{-x^{2} / 2}, x \in \mathbb{R}$. Then, the following proposition gives an evaluation of the probability that the event $\{0 \leq X \leq t\}$ occurs with $t \in[0,1]$.

Proposition 2. Let $X$ be a random variable with the standard normal distribution. For any $t \in[0,1]$, we have

$$
P(0 \leq X \leq t) \geq \frac{1}{\sqrt{2 \pi}}\left[-\frac{3}{2} t^{3}+\frac{1}{8} t^{4}+8 \gamma\left(3, \frac{t}{2}\right)+t\right],
$$

where $\gamma(a, x)=\int_{0}^{x} t^{a-1} e^{-t} d t$ is the standard incomplete gamma function. 
Proof. By applying Proposition 1 with $x \in[0,1]$ and $a=-x / 2$, we have

$$
e^{-x^{2} / 2} \geq-\frac{1}{2} x^{2}(1-x)+x^{2}\left(e^{-x / 2}-1\right)+1
$$

which implies that

$$
\begin{aligned}
P(0 \leq X \leq t) & \geq \frac{1}{\sqrt{2 \pi}}\left(-\frac{1}{2} \int_{0}^{t} x^{2} d x+\frac{1}{2} \int_{0}^{t} x^{3} d x+\int_{0}^{t} x^{2} e^{-x / 2} d x-\int_{0}^{t} x^{2} d x+t\right) \\
& =\frac{1}{\sqrt{2 \pi}}\left[-\frac{3}{2} t^{3}+\frac{1}{8} t^{4}+8 \gamma\left(3, \frac{t}{2}\right)+t\right] .
\end{aligned}
$$

The proof of Proposition 2 ends.

In Proposition 2, it is intriguing to see how polynomial and gamma functions appear to bound a probability function.

Figure 2 illustrates the lower bound in Proposition 2 by showing the curve of the function

$$
F(x)=P(0 \leq X \leq x)-\frac{1}{\sqrt{2 \pi}}\left[-\frac{3}{2} x^{3}+\frac{1}{8} x^{4}+8 \gamma\left(3, \frac{x}{2}\right)+x\right]
$$

for $x \in[0,1]$.

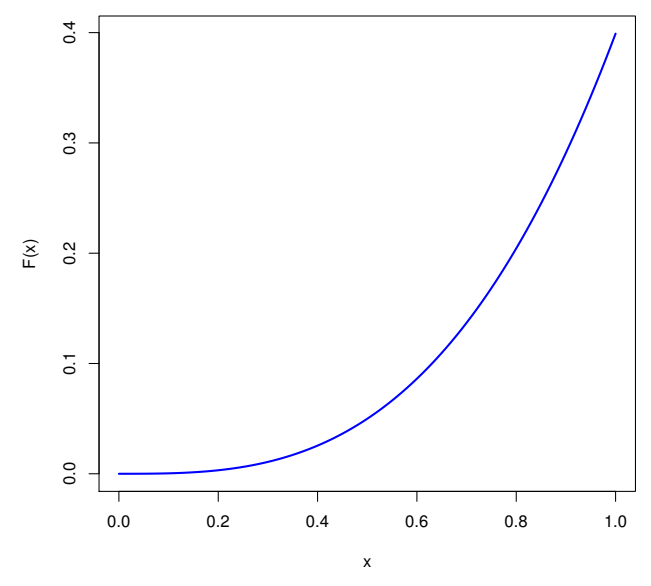

Figure 2. Curve for $F(x)$ for $x \in[0,1]$.

In Figure 2, we see that the bound is very sharp for $x \in[0,0.6]$. However, we do not claim that it is the "sharpest lower bound ever" for $P(0 \leq X \leq t)$, but just an interesting application of our main result. 
Remark 5. Proposition 2 can be used to bound the cumulative distribution function of $X$; Since, for $t \in[0,1], P(X \leq t)=1 / 2+P(0 \leq X \leq t)$, we have

$$
P(X \leq t) \geq \frac{1}{\sqrt{2 \pi}}\left[-\frac{3}{2} t^{3}+\frac{1}{8} t^{4}+8 \gamma\left(3, \frac{t}{2}\right)+t\right]-\frac{1}{2},
$$

3.2. Bound of the Kummer beta function. Proposition 1 can be used for approximation purposes. For instance, let us consider the Kummer beta function defined by

$$
\mathcal{I}(a, \alpha, \beta)=\int_{0}^{1} e^{a x} x^{\alpha-1}(1-x)^{\beta-1} d x
$$

with $a \in \mathbb{R}, \alpha>0$ and $\beta>0$. This function has found numerous applications in probability and statistics. See [4-7] in this regard, and it remained complicated to evaluate with simple functions. Thanks to Proposition 1, the following result can be proved.

Proposition 3. Let $\mathcal{I}(a, \alpha, \beta)$ be the Kummer beta function as defined by (3). For any $a \in \mathbb{R}$, we have

$$
\operatorname{sign}(a) \mathcal{I}(a, \alpha, \beta) \leq \operatorname{sign}(a)\left[a B(\alpha+1, \beta+1)+\left(e^{a}-1\right) B(\alpha+2, \beta)+B(\alpha, \beta)\right],
$$

where $B(\alpha, \beta)=\int_{0}^{1} x^{\alpha-1}(1-x)^{\beta-1} d x$ is the standard beta function.

Proof. The proof is a direct application of Proposition 1 and basic integral properties:

- For $a>0$, by applying Proposition 1, we get

$$
e^{a x} \leq a x(1-x)+x^{2}\left(e^{a}-1\right)+1
$$

which implies that, after some developments,

$$
\mathcal{I}(a, \alpha, \beta) \leq a B(\alpha+1, \beta+1)+\left(e^{a}-1\right) B(\alpha+2, \beta)+B(\alpha, \beta)
$$

- For $a<0$, by also applying Proposition 1, we get the reverse inequality:

$$
\mathcal{I}(a, \alpha, \beta) \geq a B(\alpha+1, \beta+1)+\left(e^{a}-1\right) B(\alpha+2, \beta)+B(\alpha, \beta),
$$

The proof of Proposition 3 is complete.

Thus, thanks to Proposition 3, the standard beta function can be used to evaluate quite precisely the Kummer beta function.

Other applications can be given; this study just opens a door for more in this direction. 


\section{REFERENCES}

[1] J. Bae, Optimal polynomial bounds for the exponential function, Math. Inequal. Appl. 3 (2013), 763-782. https://doi.org/10.7153/mia-16-58.

[2] I.S. Gradshteyn, I.M. Ryzhik, Table of integrals, series, and products, Academic Press, New York, 2007.

[3] S.-H. Kim, Densely algebraic bounds for the exponential function, Proc. Amer. Math. Soc. 135 (2007), 237-241. https://www.jstor.org/stable/20534567.

[4] D.K. Nagar, A.K. Gupta, Matrix-variate Kummer-beta distribution, J. Aust. Math. Soc. 73 (2002), 11-26. https://doi.org/10.1017/S1446788700008442.

[5] D.K. Nagar, E. Zarrazola, Distributions of the product and the quotient of independent Kummer-beta variables, Sci. Math. Japon. 61 (2005), 109-117.

[6] K.W. Ng, S. Kotz, Kummer-gamma and Kummer-beta univariate and multivariate distributions. Research Report, 84, Department of Statistics, The University of Hong Kong, Hong Kong, (1995).

[7] R.R. Pescim, G.M. Cordeiro, C.G.B. Demétrio, E.M.M. Ortega, S. Nadarajah, The new class of Kummer beta generalized distributions. SORT, 36 (2012), 153-180. 